\section{A Comparative Analysis upon Mobile Healthcare System and Services: A Case Study of Rural India}

\begin{abstract}
Mobile healthcare provision in the home environment presents many difficulties. Mobile Healthcare is a term used for the practice of medicine and public health supported by mobile phones. It is most generally utilized as a part of the reference to using mobile communication devices for example mobile phones, tablet and computer PDAs. A wireless sensor network with a large number of sensor hubs can be utilized as effective powerful tool for gathering data in mobile healthcare situations. It is an independent sensor to monitor the physical or natural condition. This paper addresses different algorithms and techniques of the Mobile Healthcare System.

Mobile technologies are progressively growing in developing countries like India. There have been a few new researches and improvements in this space. These days, mobile is becoming an important ICT instrument in urban locales, as well as in remote and rural areas. The rapid progress in the technologies, usability and the falling expenses of devices, make the mobile an appropriate and adaptable tool to bridge the digital divide. Mobile phone ownership in India is growing rapidly, six million new mobile subscriptions are included every month and one in five Indian's will possess a phone by the end of 2007. By the finish of 2008, three quarters of India's population will be covered by a mobile network. A large portion of these new "mobile citizens" live in poorer and more rural areas with scarce infrastructure and facilities, high illiteracy levels, low PC and internet penetration.

The availability of minimal effort mobile phones and the already broad coverage of GSM systems in India is a huge opportunity to give benefits that would trigger advances and enhance individual's lives. This paper investigates the present status of Mobile based Health Care systems in various countries, deficiencies in Primary Health Care Management in country India, and the potential answer to fill it with the empowering of Mobile Web advancements for Primary Health Care management.
\end{abstract}

\section{Introduction}

This paper provides an outline of the key application ranges of mobile telephony in health programs, popularly named as mHealth, particularly in primary healthcare in rural India. It additionally highlights the basic ranges that require further research and development in areas where mechanical and operational challenges systematically impede the health sector taking advantages of mHealth [1].

Rapid growth of mobile telephony, together with the low acceptability of other data and communication technologies (ICTs), has encouraged developing countries to apply portable communication in taking care of specific issues in the health sector which has long established the need for appropriate ICT interventions. In India, Telecom Regulatory Authority of India (TRAI) maintains and publishes data on telephone subscriptions. According to TRAI,

\section{Journal of}

Emergency Medicine \& Critical Care

\author{
Mohammad Chand Jamali* \\ Department of Health \& Medical Sciences, Khawarizmi International \\ College, United Arab Emirates \\ *Address for Correspondence: \\ Mohammad Chand Jamali, Department of Health \& Medical Sciences, \\ Khawarizmi International College, 25669, Abu Dhabi, United Arab \\ Emirates, E-mail: mjamali68@gmail.com \\ Submission: 25 January, 2017 \\ Accepted: 20 April, 2017 \\ Published: 29 April, 2017 \\ Copyright: (๑) 2017 Jamali MC. This is an open access article distributed \\ under the Creative Commons Attribution License, which permits \\ unrestricted use, distribution, and reproduction in any medium, provided \\ the original work is properly cited.
}

India has approximately 858 million mobile subscribers by the end of July this year, compared to 652 million the earlier year. In a nation of 1.21 billion individuals, this yearly development of $32 \%$ means $35 \%$ teledensity in rural India. Even though this coverage appears to be low, mobile telephony has secured a significantly higher number of rural households. The mobile phone coverage is as of now comparable to that of prominent media like radio and television, which have been utilized for spreading health awareness for a long time now. For a long time to come, mobile telephony will remain perhaps the most available ICT media in rural India.

According to the ITU, the aggregate number of mobile users worldwide starting late 2006 was around 2.7 billion and the quantity of Internet clients was just above 1.1 billion. This implies in any event there is $23.6 \%$ of world population (and no less than $22.2 \%$ of developing countries populations) who already have mobile phones but are not yet utilizing the Internet. Mobile services are rapidly developing as the new frontier in changing government and making it significantly more accessible and citizen-centric by extending the advantages of remote delivery of government services and information to those who can't or unwilling to get to open administration services through the Internet or who just prefer to use mobile devices. In principle, numerous government services can be presently made accessible on a $24 \times 7 \times 365$ basis at wherever in the world secured by mobile networks, which today means almost everywhere. Approximately 50\%-60\% of government services including Primary Health Management can be conveyed by means of mobile channel [2].

Primary Health Care Services using Mobile Devices guarantee enhanced access to primary healthcare and its door-keeping capacity prompts less hospitalization, and less shot of patients being subjected to inappropriate health interventions.

\section{Mobile/Portable Technology for Health Care}

Among the numerous ICT options available to government to enhance the proficiency and effectiveness of its delivery process of primary health care, mobile and wireless technologies offer some exciting opportunities for a minimal effort, high achieve benefit [36]. 
There is solid evidence that mobile technologies could be instrumental in tending to moderate reaction rates of government to citizen requests, poor access to administrations, especially for low-income and underestimated populations in underserviced rural areas. In addition, mobile technologies offer significant opportunities for enhancing the back-office operations of govt. Moreover, numerous primary healthcare centers situated in the rural areas do not have any electronic systems at all, and keep on operating paper- based systems, bringing about patient records being kept by patients themselves. The effect of the use of different systems is that it is difficult and costly to build up a national review of patient statistics. On a more fundamental level, it is extremely difficult for individual establishments inside the healthcare sector to share data between each other. One of the clearest examples of this is to be found in the sharing of patient laboratory outcomes. As of now in many occasions, this alone happens through manual exchange. Numerous vendors of Cellular phones started to embed a variety of eHealth administrations in Mobiles.

Various Organizations have been working at different projects for improving the role of ICT in Health care. UK-based Loughborough University's engineers have entered upon as association with experts of India to build up a unique mobile phone health monitoring system. The system, which was initially unveiled in 2005, utilizes a mobile phone to transmit a person's fundamental signs, including the unpredictable electrocardiogram (ECG) heart signal, to a hospital or clinic any place in the world. Professor Bryan Woodward and Dr. Fadlee Rasid from the Department of Electronic and Electrical Engineering at the Loughborough University have build up this mobile phone monitoring system.

Presently the system can exchange the sign relating to the ECG, blood pressure, oxygen saturation and blood glucose level. Presently the UK-India Education and Research Initiative (UKIERI) have awarded Professor Woodward a grant to further develop this mobile phone monitoring system. They have tied up with the Indian Institute of Technology Delhi (IIT Delhi), the All India Institute of Medical Sciences and Aligarh Muslim University and London's Kingston University, to additionally build up the system.

The research team is aiming to scale down the system, through outlining sensors and smaller than normal-processors that are small enough to be conveyed by patients and in the meantime get biomedical data. The system of sensors would be connected through a modem to mobile networks and the Internet, and to a hospital computer. At that point, doctors can utilize this gadget to remotely screen patients experiencing from chronic diseases, similar to heart disease and diabetes, which plagues millions over the world. The UK government will elevate the device to enhance the effectiveness of healthcare delivery. In India, the project will link clinics and regional hospitals in remote ranges to focuses of excellence. The clinical trials of the system will take place in the UK and India in the following three years.

\section{Accomplishments and Challenges}

Mobile communications like SMS and calls are being utilized as a part of numerous public service delivery projects across India by different government departments, as a major aspect of their
e-Government activities. Specifically, the condition of states Gujarat and Chhattisgarh have effectively utilized SMS to intimate the beneficiaries of the lifting of nourishment supplies by the reasonable value shops, operating under the Public Distribution System (PDS). In 2009, Bihar executed the SMS Based Monitoring System to encourage government officers to send day by day reports through SMS on the key accomplishments in ten most critical government schemes. These activities underline that the public officers and beneficiaries at the community level can adopt mobile communications.

Notwithstanding these accomplishments in different social and public sectors, advances in mHealth in India is fairly constrained. Most mHealth tasks in India began their trip around 2008. Inspite of the guarantees with information gathering using mobile devices, the tests are yet to decisively establish the acceptance and possibility of this strategy for huge-scale deployment. Specifically, a sizeable number of ASHAs in northern India have demonstrated their restricted acceptance of information accumulation using SMS and mobile structures. This process needs additionally research and innovative work. Few projects in India have added to the advancement of a more full comprehension of behavior change forms encouraged by mobile communication.

Challenges in the selection of mHealth are different. There are technological difficulties in info shows, display, transfer and preparation of data in English and Indic languages (particularly for individual with different levels of education), security and combination with medical devices. Traditional health research foundations need ability to create mHealth interventions that require multidisciplinary methodologies of behavioral, therapeutic, business and computer sciences. The public health departments in central and state governments also lack the capacity to design and implement mHealth on the ground.

\section{M-Health: A Paradigm Shift in Healthcare Services}

E-Health which is characterized as electronic trade of health care data or information across over associations has happened in three ranges: business, clinical, and consumer. Business e-Health joins the authoritative and money related parts of delivering health care and is the most created. Consumer's e-Health is apparent in the form accessibility of healthcare information from internet resources is also well developed. Clinical e-Health has been the slowest to grow; be that as it may, it offers openings in effectiveness and enhanced patient care. Mobile e-Health or $\mathrm{m}$-Health comprehensively envelopes the utilization of media transmission and sight and sound innovations coordinated within progressively mobile and wireless health care delivery systems [7]. M-Health Summit of the Foundation (2010), characterized, m-Health as the "the delivery of healthcare services administrations by means of mobile communication devices". $\mathrm{m}$-Health has been considered and distinguished as one of the five key patterns reshaping the eventual fate of health care viz. new responsibility ideas reshaping healthcare delivery models, changing channels, virtual care models and telehealth, vertical integration, diversification and the rise of health care aggregates and last however not the minimum is the $\mathrm{m}$-Health. The mobile, smart phones and tablets have changed the communications, commerce, and stimulation through better administration, enabled 
consumers by changing the method for getting to data and doing transactions. Mobile devices which can be utilized as a part of $\mathrm{m}$-Health incorporate laptops, tablets, mobile phones, smart phones, palmtops, notebook, net book, etc. There are two assortments of mobile phones - voice mobile phone and advanced smart phones. India has 750 million (11\%) versatile memberships out of 6.8 billion mobile subscriptions around the world. Among the different smart mobile devices, advanced mobile phone had most extreme $60.1 \%$ market share rather than tablet with $10.7 \%$. As Indicated by Canalys, tablet market will grow two times quicker than advanced mobile phone market worldwide in the following five years (2011-2016).

India can be considered as a youthful nation as its $65 \%$ of the population is beneath the age of 35 years. Administration of India's spending on health care is under $2 \%$ of GDP, which is most reduced around the world. India has 15\% of guaranteed and $85 \%$ of the uninsured population. There is a dispersion dissimilarity of healthcare services in India with $80 \%$ of health care services administrations amassed in urban zones as opposed to provincial areas. India has geological attributes with numerous remote ranges out of reach and inaccessible to reasonable healthcare. Every one of these issues makes a perfect ideal opportunity for $\mathrm{m}$-Health to take care of the issue. The mobile phone entrance in India is $72 \%$ with $13 \%$ Smart telephone infiltration. The web access through mobile devices stays $26 \%$ in the world (US Census Bureau Internet World Stat, 2014). The four most often revealed $\mathrm{m}$-Health applications are health call focuses, crisis toll free telephone utilities, control crisis and disasters and mobile telemedicine. Healthcare delivery in poor nations is a key challenge that $\mathrm{m}$-Health is very much ready to address. M-Health is not an optional choice today, rather a phenomenal test and an unquestionable requirement for a major nation like India. One of the undesirable outcomes of present day healthcare has been loss of correspondence and developing separation amongst doctors and patients. M-Health today is a little part of the health care landscape in India, yet its future is by all accounts emotional. The m-Health market in India is anticipated to be of the extent of US 1 billion by the year 2020 A.D. This market should be made out of (i) m-Health advertise focusing on individual patients - US\$ 800 million and (ii) m-Health market focusing on physicians, hospitals of the size - US\$ 200 million. UK-based Loughborough University's engineers have entered upon an organization with specialists of India to build up an exceptional mobile phone health monitoring system. The system, which was initially uncovered in 2005 , utilizes a mobile phone to transmit a man's indispensable signs, including the intricate electrocardiogram (ECG) heart signal, to a hospital or clinic anyplace in the world. Sehat Saathi, a country telemedicine system is being developed at Media Lab Asia research hub at IIT Kanpur.

It can be utilized to stretch medical care to patients in the remote parts of the nations. The model accommodates for front-end contact through a reasonably prepared non-medical professional, back end support from specialists, pathologists and other health experts for determination and treatment, utilization of digital innovation technology to accomplish objects; and dispersal of information on health and disease through advanced means. Some key m-Health arrangements which may end up being emotional in Indian scenario are diabetes checking, asthma observing, medication compliance, data dissemination, e-Prescriptions, mobile imaging, non specific remote checking and resource administration. CDAC, Electronics City, Bangalore has started the advancement of Mobile Based Primary Health Care Management System for arrangement for improvement of administration of Primary Health Care specifically in the country and urban slums of India.

\section{Country Health: Expanding Access in a One-To-Many Approach}

Country territories confront the greatest deficiencies of medicinal services limit in India. Although two-thirds of the population lives in rural areas, only one fourth of healthcare infrastructure and man power is situated in provincial areas. This circumstance is probably not going to be enhanced soon by government spending or customary private healthcare. The expenses are just too high. mHealth can possibly supply cost-effective care to provincial India.

Not at all like a more urban setting, rural people are probably not going to have the capacity to get to mHealth administrations at the family unit level $[5,6]$. While every one of the $(94 \%)$ of urban family units have a mobile phone, just about half (58\%) of rural households have phone access. Reliable energy to charge telephones can likewise be an issue. Not every mobile phone will be smart phones equipped for information trade either and. Also, uneducated people in rural communities may have issues utilizing mHealth applications.

Grassroots systems in provincial India are an effective solution to the issue of innovation get to. A standout amongst the most surely understood models is ITC e-Choupal which offers country web access to farmers at the town level, who are frequently helped by a peer capable of utilizing the system. The administration permits farmers to get to agricultural information and also market prices, enabling them to boost production and receive the best possible price for their goods. Community-driven networks like ITC e-Choupal, which coordinate country attitude and technology, should be the basis of any rural mHealth activity.

For mHealth to prevail in rural regions a community-based technique will be required. Dissimilar to more typical mHealth associations that set up a connection between a remote carrier and a healthcare provider, country mHealth may require a more intricate model. A formerly displayed demonstrate, intended to address a varitey of mHealth barriers in rural areas, suggested a combination of utilizing human resources, enhancing supply chains, giving small scale protection and utilizing low-data transmission applications. Resources must be shared to bring down per client expenses and foster community involvement.

\section{Conclusion}

The Primary Health Care methodology seems to be a right intervention in terms of basic preventive methods however it needs to be upheld by different strategies also to close the holes. Primary health care is introduced by the Alma-Ata announcement as basic health care based on practical, scientifically sound and socially adequate techniques and innovation, which is all around available to individuals, family and the community through their full cooperation and at the cost they can manage. The Primary Health Care can be made straightforward and effectively available by the usage of "Mobile based Primary Health Care Management System". In this paper, the subsystems of the proposed system have been brought out. 
Citation: Jamali MC. A Comparative Analysis upon Mobile Healthcare System and Services: A Case Study of Rural India. J Emerg Med Critical Care 2017;3(1): 4

ISSN: $2469-4045$

Mediation studies and tasks in developing nations have exhibited the applications and adequacy of mHealth in different health areas in resource-poor settings. In India, all the more such medications are important to configure better mHealth programs for the concerned group. Indigenous innovative work in key technology areas, and also innovation exchange from different nations, will help in explaining technological weaknesses. Interests as help give and capital can goad research and advancement around in this area. mHealth in its current form is exploiting the second generation mobile telephony which will continue to dominate rural health for several years to come. The third era of mobile telecommunication has been seeing tepid reaction both in rustic and urban areas. As mobile broadband makes its advances in provincial areas, mHealth can increase the value to the health division.

\section{References}

1. Arshad U, Mascolo C, Mellor M (2003) Exploiting mobile computing in health-care.

2. Han D, Park S, Kurkuri S (2006) An evolving mobile e-health service platform.

3. (2012) Progressions-The third place: Health care everywhere. EYGM Limited.

4. Patel N (2005) Evaluating the role of primary health centers in India. Express Health care Management 16-31.

5. Murthy R. Mobile based Primary Health Care System for Rural India.

6. Patil AV, Somasundaram KV, Goyal RC (2002) Current health scenario in rural India. Aust J Rural Health 10: 129-135.

7. Ramachandran D, Canny J, Das PD, Cutrell E (2010) Mobile-izing health workers in rural India. Proceedings of the $28^{\text {th }}$ international conference on Human factors in computing systems (CHI '10). ACM New York, NY, USA pp. 1889-1898. 